UNIVERSIDADE TECNOLÓGICA FEDERAL DO PARANÁ DEPARTAMENTO ACADÊMICO DE ENGENHARIA DA PRODUÇÃO MBA EM GESTÃO DE NEGÓCIOS COM ÊNFASE EM GERENCIAMENTO DE PROJETOS

JORGE LUIS SANTOS

O USO DO RELATÓRIO A3 COMO FERRAMENTA DE IMPLEMENTAÇÃO DA FILOSOFIA LEAN NA GESTÃO DE EMPRESAS DO SETOR DE CONSTRUÇÃO CIVIL

MONOGRAFIA DE ESPECIALIZAÇÃO 
JORGE LUIS SANTOS

O USO DO RELATÓRIO A3 COMO FERRAMENTA DE IMPLEMENTAÇÃO DA FILOSOFIA LEAN NA GESTÃO DE EMPRESAS DO SETOR DE CONSTRUÇÃO CIVIL

Trabalho de Conclusão de Curso apresentada como requisito parcial à obtenção do título de Especialista em Gestão de Negócios com Ênfase em Gerenciamento de Projetos da Universidade Tecnológica Federal do Paraná - Campus Londrina.

Orientador: Prof. Dr. Marco Antônio Ferreira 
Ministério da Educação

UNIVERSIDADE TECNOLÓGICA FEDERAL DO PARANÁ

Campus Londrina

DAENP - Departamento Acadêmico de Engenharia da Produção

\section{TERMO DE APROVAÇÃO}

O USO DO RELATÓRIO A3 COMO FERRAMENTA DE IMPLEMENTAÇÃO DA FILOSOFIA LEAN NA GESTÃO DE EMPRESAS DO SETOR DE CONSTRUÇÃO CIVIL

por

JORGE LUIS SANTOS

Este Trabalho de Conclusão de Curso de Especialização foi apresentado em 06 de dezembro de 2019 como requisito parcial para a obtenção do título de Especialista em Gestão de Negócios com Ênfase em Gerenciamento de Projetos. O(a) candidato(a) foi arguido(a) pela Banca Examinadora composta pelos professores abaixo assinados. Após deliberação, a Banca Examinadora considerou o trabalho aprovado.

\begin{tabular}{c}
\hline Prof. Dr. Marco Antônio Ferreira \\
Prof.(a) Orientador(a) \\
\hline Prof. Dr. Bruno Samways dos Santos \\
Membro titular \\
\hline Prof. Dr. Rafael Henrique Palma Lima \\
Membro titular
\end{tabular}

- O Termo de Aprovação assinado encontra-se na Coordenação do Curso - 


\section{RESUMO}

SANTOS, Jorge Luis; FERREIRA, Marco Antônio. O uso do relatório A3 como ferramenta de implementação da filosofia lean na gestão de empresas do setor de construção civil. 2019. 18 páginas. Monografia (Especialização em Gestão de Negócios com Ênfase em Gerenciamento de Projetos) - Universidade Tecnológica Federal do Paraná. Londrina, 2019.

Diante do cenário econômico e a consequente crise do setor da construção civil, muitas empresas que vinham perdendo espaço no mercado imobiliário se viram obrigadas a rever os seus modelos de negócio e processos afim de transporem a crise e se consolidarem de forma estruturada para a retomada econômica do setor da construção civil. Perante à esse desafio que as empresas da construção civil passaram a implementar a filosofia Lean de forma sistêmica e estratégica em seus negócios e processos executivos, elevando, dessa forma, a sua capacidade produtiva e a qualidade do produto.

O relatório A3 é uma ferramenta com muito potencial de utilidade para as organizações na solução de problemas, desde sua análise até a proposta de soluções de forma clara e sucinta. Quando tem a sua implementação de forma apropriada, sua abordagem pressiona a empresa em direção à uma visão sistêmica da melhoria dos seus processos ao invés da otimização pontual. Assim sendo, a implementação dessa ferramenta aliada à filosofia Lean nas empresas do setor da construção civil visa a melhoria sistêmica das organizações afim de se consolidarem para a retomada do setor.

Palavras chave: A3, Construção civil, Lean, Linha de Balanço, Processos. 


\begin{abstract}
SANTOS, Jorge Luis; FERREIRA, Marco Antônio. Using the A3 report as a tool for implementing the lean philosophy in the management of construction companies. 2019. 18 pages. Monograph (Especialization in MBA in Business Management with an emphasis on Project Management) - Federal Technology University - Paraná. Londrina, 2019.
\end{abstract}

Faced with the economic scenario and the consequent crisis in the construction sector, many companies that had been losing ground in the real estate market were forced to review their business models. This also includes processes in order to overcome the crisis and consolidate in a structured way for the economic recovery of the construction sector. Faced with this challenge, construction companies began to implement the lean philosophy in a systemic and strategic way in their business and executive processes, thus increasing their production capacity and product quality.

The A3 report is a very useful tool for organizations to solve problems, from analysis to proposing solutions clearly and succinctly. When properly implemented, its approach pushes the company toward a systemic view of process improvement rather than timely optimization. Thus, the implementation of this tool combined with the lean philosophy in companies in the construction sector aims at systemic improvement of organizations in order to consolidate themselves for the recovery of the sector.

Key-words: A3, Construction Sector, Lean, Line of Balance, Processes. 
1 INTRODUÇÃO

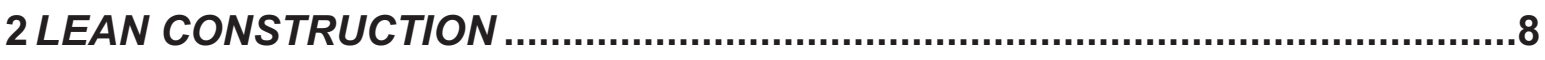

3 FERRAMENTA A3

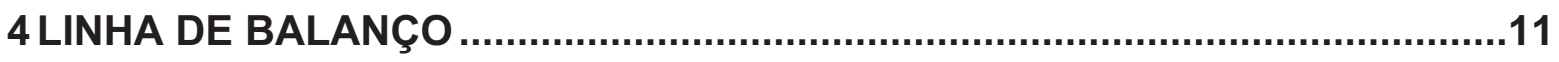

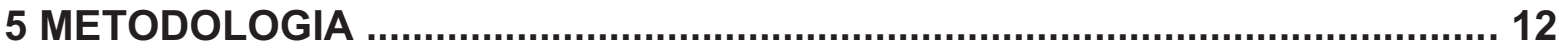

6 CONCLUSÃO

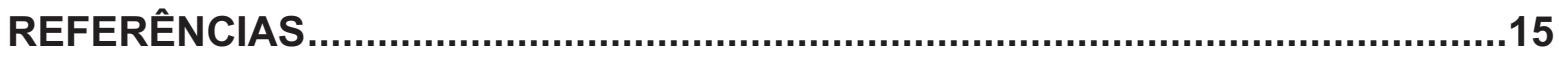

ANEXO A - CERTIFICADO DE ACEITE DO ARTIGO ......................................17

ANEXO B - CERTIFICADO DE PUBLICAÇÃO DO ARTIGO NO LIVRO DA EDITORA CONHECIMENTO LIVRE 


\title{
O uso do relatório A3 como ferramenta de implementação da filosofia Lean na gestão de empresas do setor de construção civil
}

\author{
Jorge Luis Santos (UTFPR - Campus Londrina) jorgelstos@gmail.com
}

\begin{abstract}
Resumo: Diante do cenário econômico e a consequente crise do setor da construção civil, muitas empresas que vinham perdendo espaço no mercado imobiliário se viram obrigadas a rever os seus modelos de negócio e processos afim de transporem a crise e se consolidarem de forma estruturada para a retomada econômica do setor da construção civil. Perante à esse desafio que as empresas da construção civil passaram a implementar a filosofia Lean de forma sistêmica e estratégica em seus negócios e processos executivos, elevando, dessa forma, a sua capacidade produtiva e a qualidade do produto.

O relatório A3 é uma ferramenta com muito potencial de utilidade para as organizações na solução de problemas, desde sua análise até a proposta de soluções de forma clara e sucinta. Quando tem a sua implementação de forma apropriada, sua abordagem pressiona a empresa em direção à uma visão sistêmica da melhoria dos seus processos ao invés da otimização pontual. Assim sendo, a implementação dessa ferramenta aliada à filosofia Lean nas empresas do setor da construção civil visa a melhoria sistêmica das organizações afim de se consolidarem para a retomada do setor.
\end{abstract}

Palavras chave: A3, Construção civil, Lean, Linha de Balanço, Processos.

\section{Using the $A 3$ report as a tool for implementing the lean philosophy in the management of construction companies}

\begin{abstract}
Faced with the economic scenario and the consequent crisis in the construction sector, many companies that had been losing ground in the real estate market were forced to review their business models. This also includes processes in order to overcome the crisis and consolidate in a structured way for the economic recovery of the construction sector. Faced with this challenge, construction companies began to implement the lean philosophy in a systemic and strategic way in their business and executive processes, thus increasing their production capacity and product quality. The A3 report is a very useful tool for organizations to solve problems, from analysis to proposing solutions clearly and succinctly. When properly implemented, its approach pushes the company toward a systemic view of process improvement rather than timely optimization. Thus, the implementation of this tool combined with the lean philosophy in companies in the construction sector aims at systemic improvement of organizations in order to consolidate themselves for the recovery of the sector.
\end{abstract}

Key-words: A3, Construction Sector, Lean, Line of Balance, Processes.

\section{Introdução}

A indústria da construção civil no Brasil vem sofrendo enormes dificuldades oriundas da crise econômica estabelecida no país nos últimos anos, as quais pressionaram as empresas do setor a rever os seus modelos de negócio, bem como buscar maior produtividade nos seus processos construtivos.

Diante desse desafio, foi notório que o modelo de gestão das empresas da construção civil precisava ser revisto, visto que o sistema de tradicionalmente utilizado nesse setor apresentava-se como ineficaz diante dos novos desafios enfrentados. Segundo ISATTO ET AL. 
Ponta Grossa, PR, Brasil, 04 a 06 de dezembro de 2019

(2000), a maioria dos problemas que resultam em baixa eficiência e qualidade na construção civil têm origem em deficiências gerenciais. Sendo assim, eficiência empresarial está se tornando um requisito essencial para organizações de todos os tipos, em especial as do setor da construção civil para a sua manutenção pós recessão econômica.

De acordo com FAZINGA (2012), uma das ações que permitem o gerenciamento de forma mais eficaz do processo produtivo da construção civil tem sido a busca por referenciais teóricos que possibilitem representá-lo de maneira mais apropriada. Neste sentido, destaca-se o paradigma Lean Production, ou Produção Enxuta, surgido no Japão na década de 1950, que tem como principal expoente o Sistema Toyota de Produção (STP). NETO et. al. (2008) complementam que Lean Production é um sistema que visa à melhoria da produtividade de uma determinada empresa ou empreendimento. Seus princípios visam à redução de desperdícios, agregando mais valor ao seu produto ou serviço.

Para a filosofia Lean todo problema pode e deve ser resolvido em uma única folha de papel. É dessa forma que surge o relatório $A 3$, como uma ferramenta de gestão que busca identificar e propor, a partir da sua implementação, a resolução de problemas. De acordo com SHOOK (2008) é importante que todos aqueles que participam direta ou indiretamente do problema possam enxerga-lo de uma mesma lente. Dado isso, o relatório A3 é uma ferramenta estritamente visual, sendo possível, através da sua utilização, identificar a causa raiz dos problemas e as contramedidas possíveis para soluciona-los. Ou seja, é utilizada como um guia sistematizado de solução de problemas através de um processo rigoroso, documentando os problemas principais daquele processo e propostas de melhorias. No setor da construção civil a ferramenta, o uso da ferramenta se dá para as empresas revejam seus processos afim de se tornarem mais competitivas frente à retomada do crescimento do setor com base nos princípios da filosofia de gestão Lean.

\section{Lean Construction}

A partir dos anos 90 surgiu um novo referencial teórico para a gestão de processos na construção civil, com o objetivo de adaptar alguns conceitos e princípios gerais da área de Gestão da Produção às peculiaridades do setor. Este esforço tem sido denominado de Lean Construction, por estar fortemente baseado no paradigma da Lean Production (Produção Enxuta), que se contrapõe ao paradigma da produção em massa, cujas raízes estão no Taylorismo e Fordismo.

As ideias deste novo paradigma, em realidade, surgiram no Japão nos anos 50, a partir de duas filosofias básicas, o próprio Total Quality Management (TQM) e também o Just in Time (JIT), sendo o Sistema de Produção da Toyota no Japão a sua aplicação mais proeminente (SHINGO, 1986). No que refere-se à indústria da construção civil, o surgimento do Lean Construction foi marcado pela publicação do trabalho Application of the new production philosophy in the construction industry por Lauri Koskela em 1992. A diferença básica entre a filosofia gerencial tradicional e a gestão Lean é principalmente conceitual. A mudança mais importante para a implantação do novo paradigma é a introdução de uma nova forma de entender os processos. O modelo conceitual dominante na construção civil costuma definir a produção como um conjunto de atividades de conversão, que transformam os insumos (materiais, informação) em produtos intermediários (por exemplo, alvenaria, estrutura, revestimentos) ou final (edificação). Por essa razão, é conhecido como de modelo do processo de conversão.

Este modelo apresenta, implicitamente, as seguintes características: 
- O processo de conversão pode ser subdividido em sub-processos, que também são processos de conversão;

- O esforço de minimização do custo total de um processo em geral é focado no esforço de minimização do custo de cada sub-processo separadamente;

- O valor do produto de um sub-processo é associado somente ao custo dos seus insumos.

As principais deficiências do modelo de conversão são as seguintes:

- Existe uma parcela de atividades que compõem os fluxos físicos entre as atividades de conversão (fluxos de materiais e de mão de obra), as quais não são explicitamente consideradas. Ao contrário das atividades de conversão, estas atividades não agregam valor. Em processos complexos, como é o caso da construção de edificações, a maior parte dos custos é originada nestes fluxos físicos;

- O controle da produção e esforço de melhorias tende a ser focado nos subprocessos individuais e não no sistema de produção como um todo. Uma excessiva ênfase em melhorias nas atividades de conversão, principalmente através de inovações tecnológicas, pode deteriorar a eficiência dos fluxos e de outras atividades de conversão, limitando a melhoria da eficiência global;

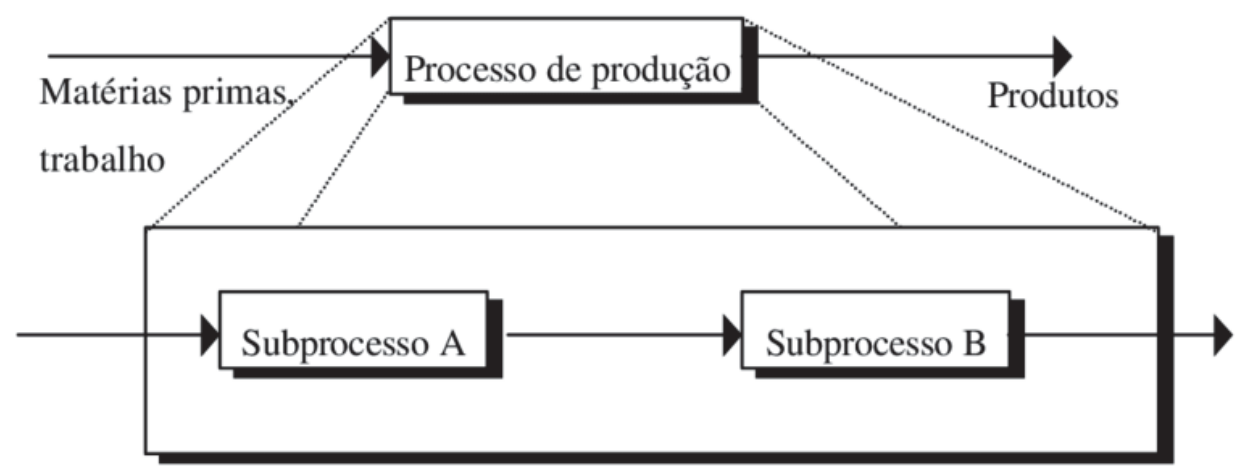

Figura 1 - Modelo do processo de conversão Fonte: ISATTO et. al. (2000)

- A não consideração dos requisitos dos clientes pode resultar na produção, com grande eficiência, de produtos que são inadequados. Neste sentido, deve-se considerar os requisitos tanto dos clientes finais como internos.

O modelo de processo do Lean Construction, por sua vez, assume que um processo consiste em um fluxo de materiais, desde a matéria prima até o produto final, sendo o mesmo constituído por atividades de transporte, espera, processamento (ou conversão) e inspeção, conforme apresentado na figura 2.

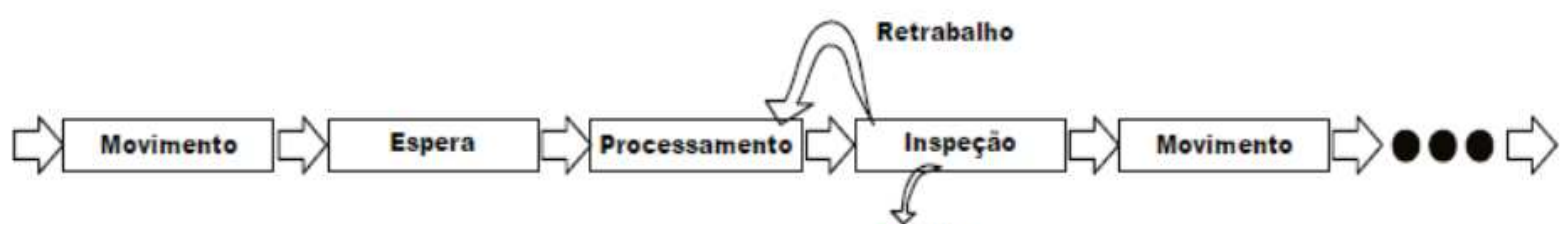

Figura 2 - Modelo do processo de conversão Lean Construction Fonte: ISATTO et. al. (2000)

As atividades de transporte, espera e inspeção não agregam valor ao produto final, sendo por 
esta razão denominadas atividades de fluxo. Nem toda a atividade de processamento agrega valor ao produto.

\section{Ferramenta $\mathbf{A} 3$}

O relatório A3 foi desenvolvido pela Toyota no começo dos anos 60 e é uma ferramenta utilizada para propor soluções para os problemas, fornece relatórios situacionais dos projetos em andamento e relatar a atividade de coleta de informações (SOBEK \& JIMMERSON, 2006). Segundo VIANA \& TORTORELLA (2014), trata-se de uma ferramenta que direciona os solucionadores de problemas a uma compreensão mais profunda do problema ou oportunidades, gerando novas ideias sobre como atacar o problema. Ademais, também proporciona o aprendizado e acúmulo de conhecimento, ajudando pessoas a aprender como aprender.

O A3 é assim chamado porque é escrito em um papel de tamanho A3 e o modelo foi inspirado no ciclo PDCA (GOSH \& SOBEK, 2002). Segundo LOYD et. al. (2010), o diferencial do pensamento $A 3$ é que não é o formato que importa, dessa forma, não existe um modelo único do relatório, mas sim uma estruturação em seções com informações básicas conforme apresentado abaixo:

- Título/Tema: indica o problema a ser abordado de forma descritiva e clara;

- Considerações iniciais: nesse item devem constar informações gerais que são essenciais para o entendimento da extensão e importância do problema;

- Estado atual: é a seção mais importante do relatório na qual são evidenciados os problemas com clareza, bem como quantificados os problemas e sua extensão (porcentagem de retrabalhos e/ou defeitos, gráficos comparativos e outros). As informações utilizadas para desenvolvimento dessa seção devem ser coletadas através de observações diretas dos processos;

- Análise: a partir do conhecimento dos problemas contidos na situação atual é que se pode ter um entendimento das causas raiz, ou seja, da origem dos problemas. Uma das maneiras de se obter o verdadeiro motivo do problema é utilizando a metodologia dos " 5 porquês";

- Estado futuro/recomendações: com o entendimento do problemas e suas contramedidas, é possível considerar imaginar como deveria ser situação ideal. Ou seja, o estado após a remoção das restrições e solução dos problemas que foram expostos anteriormente;

- Plano de ação: esboça os passos a serem seguidos até que seja atingido o estado futuro, especificando de forma clara o conteúdo, sequência, tempo e resultados. É nessa seção que é apresentado o plano de melhoria;

- Acompanhamento/Indicadores: nesse item são apresentados de forma clara e objetiva como e quando serão realizadas medições de melhorias implementadas ao sistema. Os resultados alcançados também devem estar presentes nessa seção, bem como comparativos entre antes e depois da implementação das benfeitorias. 


\begin{tabular}{|l|l|}
\hline Titulo / Tema: & Data: \\
\hline 1. Consideração iniciais (background): \\
\hline 2. Metas, Objetivos, Beneficios: \\
\hline 3. Estado Atual: \\
\\
\\
\hline
\end{tabular}

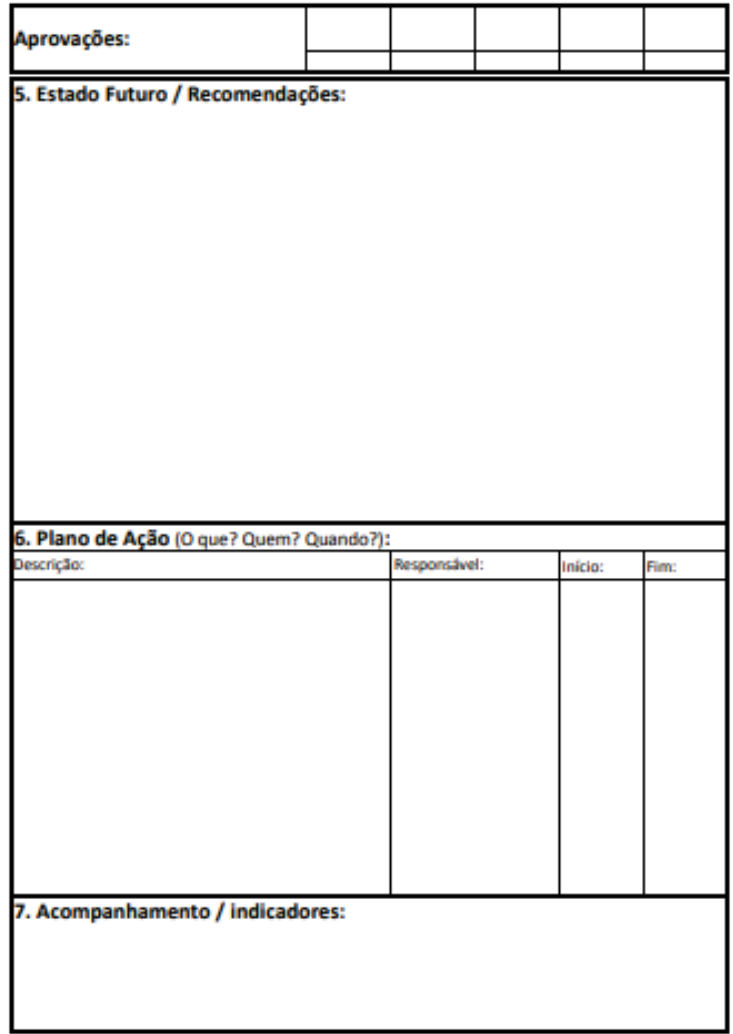

Figura 3 - Modelo de relatório A3 Fonte: Lean Institute Brasil

\section{Linha de Balanço}

Também conhecida como "diagrama de tempo-caminho", a linha de balanço é uma técnica que permite a análise a nível estratégico do cronograma, onde é possível definir e acompanhar, sem detalhamento, o plano de ataque, identificar os conflitos e gargalos de produção e acompanhar o progresso das atividades.

A linha de balanço se mostra ideal para planejamento e acompanhamento por sua praticidade, facilidade de interpretação e comunicação das informações, e é especialmente eficaz em função do caráter linear e repetitivo dos projetos de construção civil.

Segundo PADUA (2014), a linha de balanço possui outras vantagens, como menor risco no planejamento, facilidade de comparação entre os diversos planos de ataque para a obra, diminuição do tempo total do projeto e verificação rápida da viabilidade do planejamento de obra. 


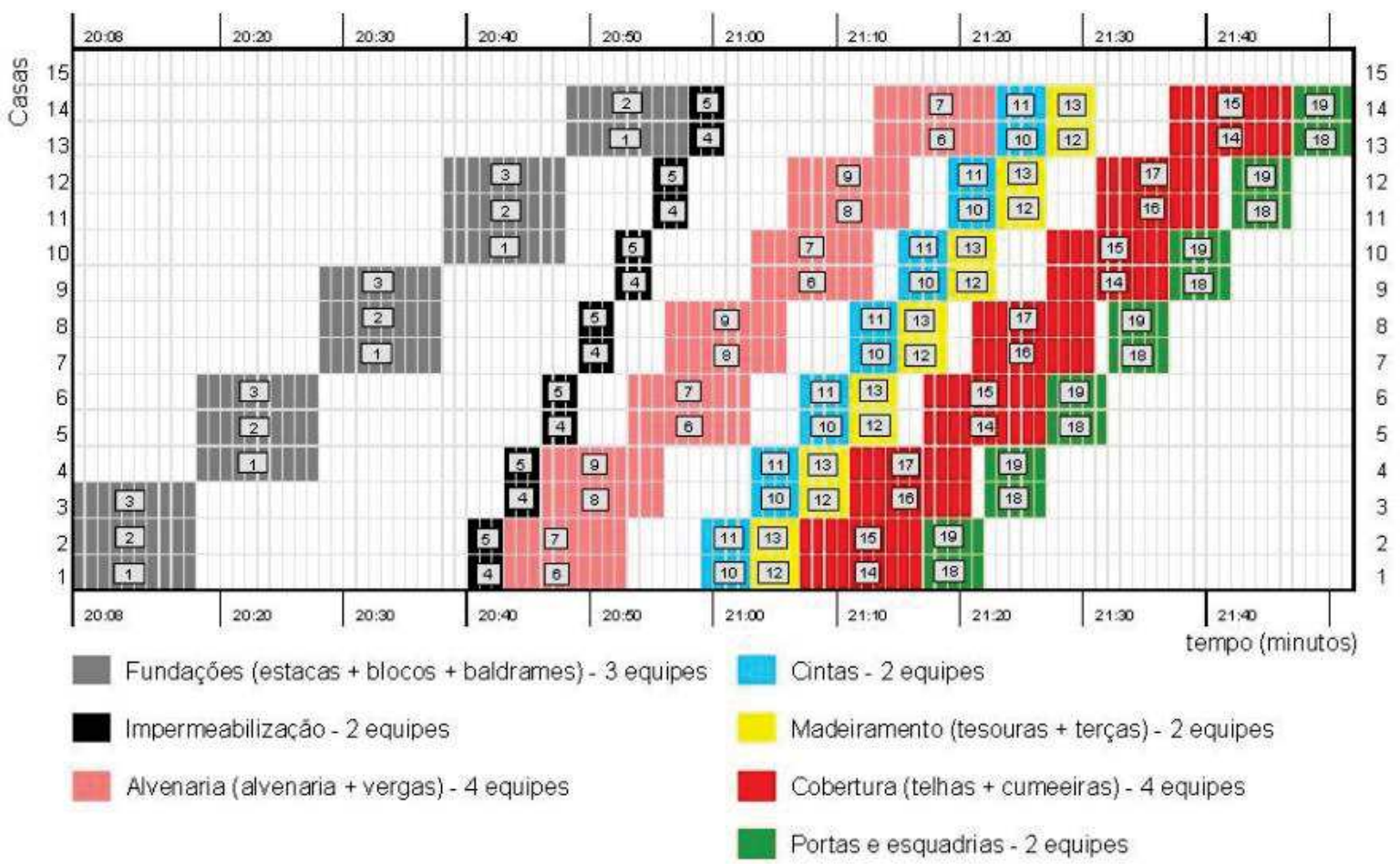

Figura 4 - Modelo de linha de balanço Fonte: SOUZA (2012)

\section{Metodologia}

Para desenvolvimento deste artigo foi utilizada como objeto de pesquisa um caso real de obra industrial com escopo $4.182 \mathrm{~m}^{2}$ com modelo de negócio turn key, ou seja, desde o desenvolvimento de projetos até a conclusão da construção do prédio de processos de uma indústria alimentícia, sendo o prazo total de trinta e uma semanas.

Para concorrência de orçamento, a equipe de planejamento da empresa construtora desenvolveu um cronograma de longo prazo num Gráfico de Gantt da maneira costumeira a ser feita para todas as outras concorrências.

Para este estudo propõe-se a aplicação o da ferramenta $A 3$ baseado nos princípios Lean Construction para redução do prazo da obra em questão. Dessa forma, tomou-se como base o cronograma de longo prazo para elaboração da primeira versão da linha de balanço, onde foi possível observar que o planejamento não havia levado em consideração as interferências executivas e sequenciamento de atividades, itens esses que são critérios para elaboração de uma linha de balanço. 


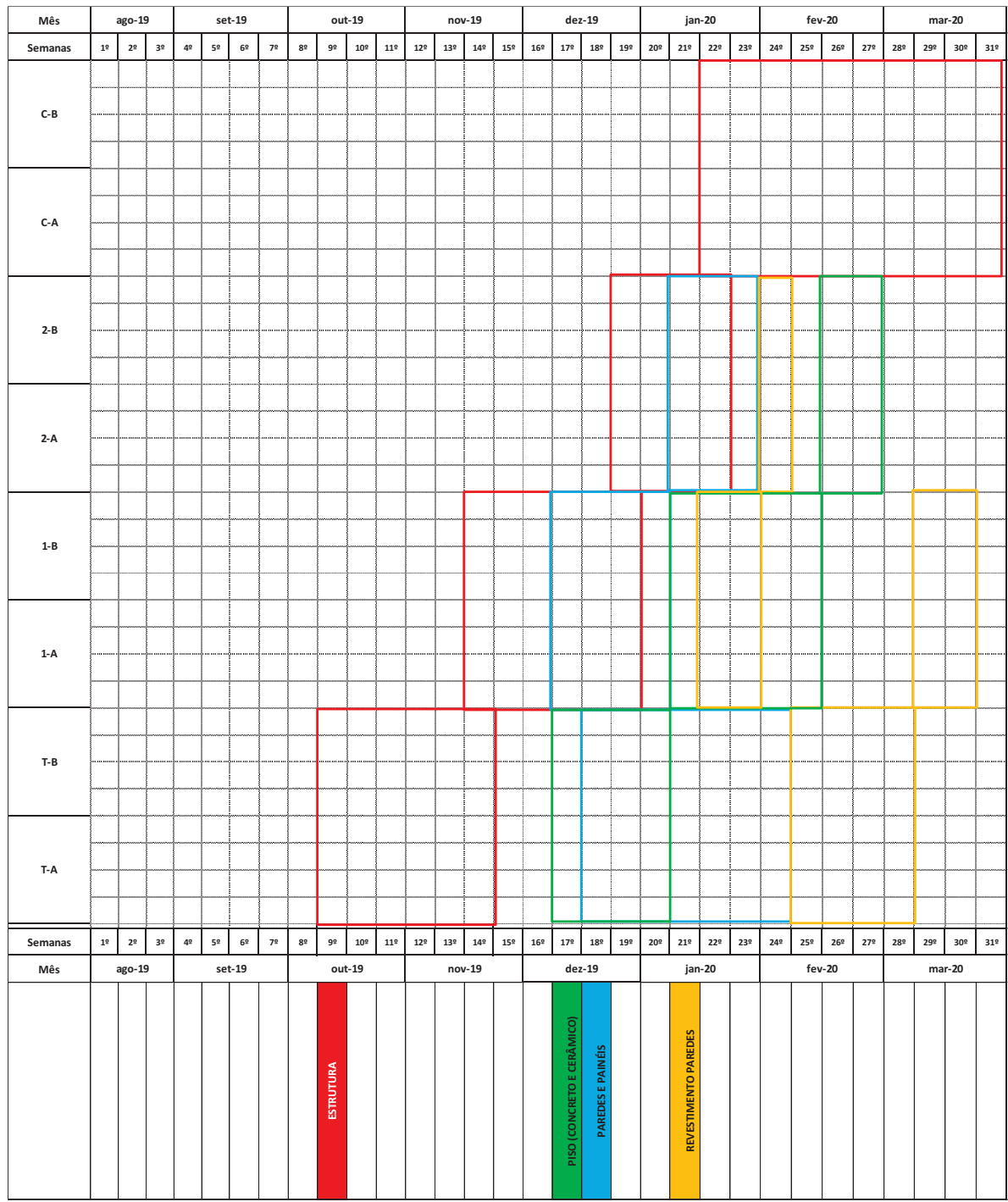

Figura 5 - Linha de balanço da obra versão inicial Fonte: o autor

Baseando-se no balanço inicial tomava-se como verdade que o prazo da obra em trinta e uma semanas era insuficiente, e que havia a possiblidade de atrasos na obra. A partir do planejamento inicial, foram propostas algumas melhorias executivas e de planejamento por meio de um relatório A3 baseando-se nos princípios Lean, conforme apresentado a seguir:

- Separação das frentes de trabalho em setor A e B reduzindo, dessa forma, o lote de trabalho;

- Mudança do sequenciamento de serviços, priorizando as atividades que permitiam a liberação de frente para outras atividades;

- Eliminação da interferência entre as atividades predecessoras e sucessoras;

- Redução do número de atividades em cada pacote trabalho, por exemplo a separação da atividade de execução do revestimento cerâmico de parede do serviço de revestimento cerâmico de piso;

- Alinhamento de ritmo dos serviços e redução do tempo de espera entre as atividades.

A partir das intervenções realizadas na linha de balanço da obra obteve-se um novo prazo 
Ponta Grossa, PR, Brasil, 04 a 06 de dezembro de 2019

para entrega, reduzindo de trinta e uma semanas para vinte e cinco semanas, validando, dessa forma, a possibilidade executiva do projeto.

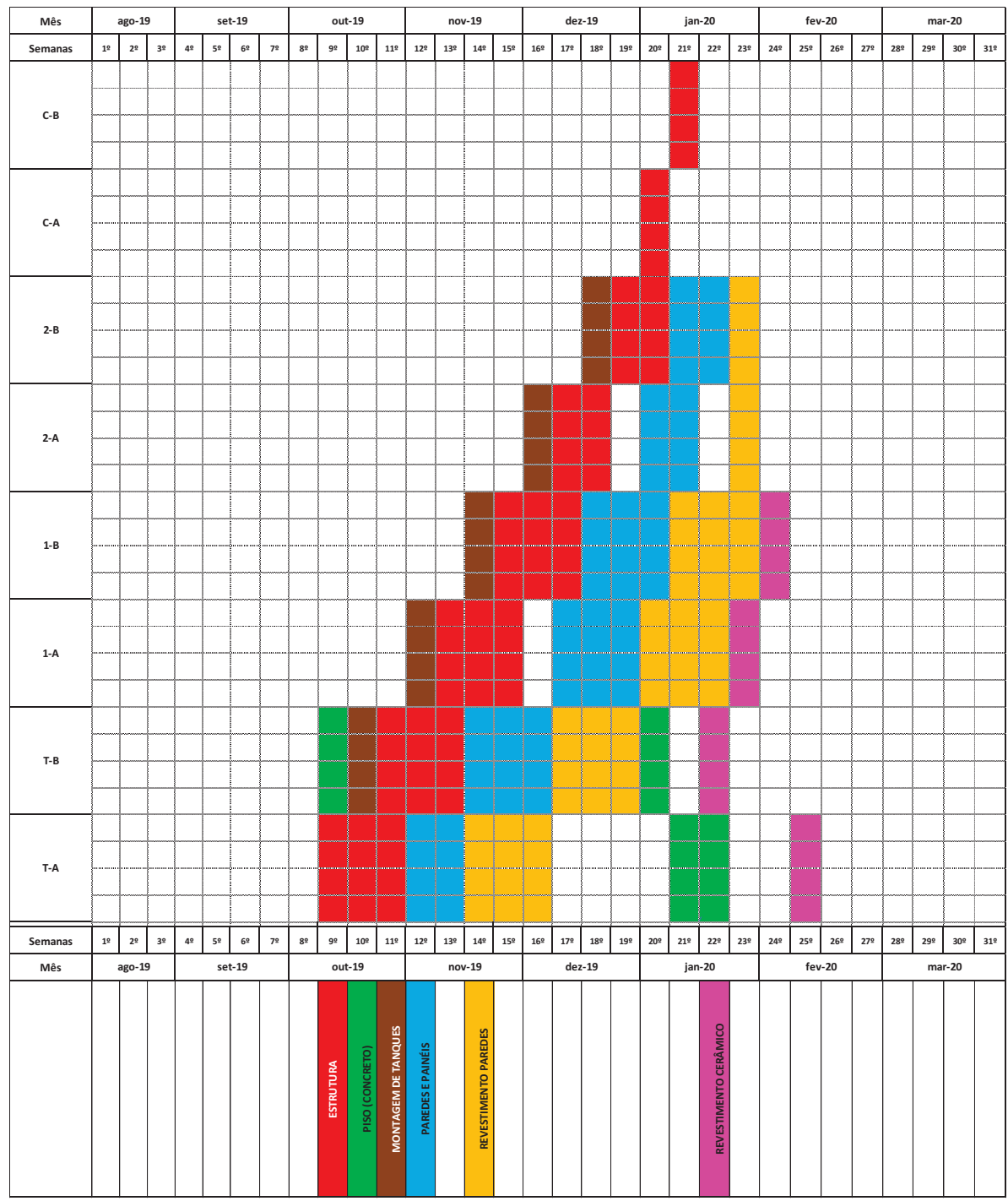

Figura 6 - Linha de balanço da obra versão final Fonte: o autor

\section{Conclusão}

Com o desenvolvimento do presente artigo pode ser evidenciado que, no âmbito da construção civil, existem várias potencialidades e dificuldades que interferem na gestão das empresas e suas atividades, tornando, dessa forma, a filosofia lean um diferencial frente ao desempenho do negócio.

O relatório $A 3$, por documentar os resultados na soluções de problemas de maneira concisa e incorporar uma completa metodologia de solução de problemas é uma ferramenta importante para promoção da melhoria de maneira rápida e eficaz.

Com a implementação da linha de balanço baseado na filosofia lean por meio da ferramenta A3 foi possível a obtenção de rápidos e expressivos resultados a partir das melhorias propostas 
no projeto em estudo, por meio do diagnósticos dos pontos críticos para execução e sua remoção.

A redução do prazo da obra era ponto chave para a viabilidade executiva do projeto, e baseado na filosofia lean foi possível confirmar a continuidade da concorrência da empresa construtora frente às demais empresas do mercado.

Assim, a partir do desenvolvimento resultados obtidos é possível inferir que toda melhoria introduzida ao processo é válida para seu aprimoramento.

\section{Referências}

<http://www.gpsustentavel.ufba.br/downloads/Producao Enxuta Engenharia de Projetos Ferramenta A Thomaz Miglio Americano da Costa.pdf> Acesso em: 03 out. 2019.

<https://www.lean.org.br/artigos/90/relatorio-a3-ferramenta-para-melhorias-deprocessos.aspx> Acesso em: 05 out. 2019.

<https://www.voitto.com.br/blog/artigo/relatorio-a3 > Acesso em: 03 out. 2018.

FARAH, Kareem. Building a competitive advantage with lean thinking. [Entrevista concedida a] Planet Lean < https://planet-lean.com/building-a-competitive-advantage-with-leanthinking/> Acesso em: 08 out. 2019.

FAZINGA, Wanessa R. Particularidades da Construção Civil para a Implantação do Trabalho Padronizado. 2012. Dissertação para obtenção do título de Mestre em Engenharia. Universidade Estadual de Londrina, Londrina.

ISATTO, Eduardo Luis et. all. Lean construction: diretrizes e ferramentas para o controle de perdas na construção civil. 2000. Porto Alegre, SEBRAE/RS.

LOYD, N. et al. Integration of A3 thinking as an academic communication standard. In: Industrial Engineering Research Conference. 2010. Cancun.

PAIXÃO ET. AL. Nível de utilização das ferramentas da filosofia Lean Construction em empresas construtoras. 2008. Fortaleza. Artigo - ENTAC.

PERARO, LUIZ EDUARDO MENEZES. O uso do kanban no transporte vertical de argamassa em edifícios de múltiplos pavimento. 2009. Trabalho de Conclusão de Curso (Graduação em Engenharia Civil) - Universidade Estadual de Londrina.

SHOOK, J. Gerenciando para o aprendizado: utilizando o processo de gerenciamento A3 para resolver problemas, promover alinhamento, orientar e liderar. 2008. Lean Institute Brasil, São Paulo.

SOBEEK, D. K.; JIMMERSON, C. A3 reports: tool for process improvement. Proceedings of industrial engineering research conference. 2004. Houston. 
PADUA, RAFAEL CRISSOSTOMO. Implementação de Práticas de Lean Construction em uma Obra Residencial em Goiânia - Estudo de Caso. 2014. Trabalho de Conclusão de Curso (Graduação em Engenharia Civil). Universidade Federal de Goiás.

VIANA, Samanta Guimarães; TORTORELLA, Guilherme. Aplicação de grupos focados e ciclos de aprendizagem na metodologia do pensamento A3: o caso de aumento da capacidade de retificação em uma siderúrgica. Universidade Federal do Rio Grande do Sul. 
ConBRepro

IX Congresso brastletro de Engenharta oe producto

ISSN: 2237.6143
CERTIFICADO

O trabalho

O uso do relatório A3 como ferramenta de implementação da filosofia Lean na gestão de empresas do setor de construção civil

sob autoria de

\section{JORGE LUIS SANTOS}

foi publicado nos anais do IX CONBREPRO.

Realizado de 04 a 06 de dezembro de 2019, no campus Ponta Grossa - PR da Universidade Tecnológica Federal do Paraná (UTFPR).

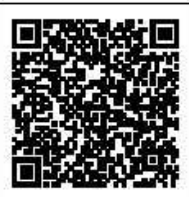

Pró. Dr. Giberto Zammar Presidente da Comissao Cientifica

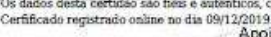




\title{
ANEXO B - CERTIFICADO DE PUBLICAÇÃO DO ARTIGO NO LIVRO DA EDITORA CONHECIMENTO LIVRE
}

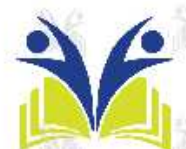

Editora Conhecimento Livre

\section{CERTIFICADO DE PUBLICAÇÃO}

Data: $23 / 09 / 2020$

Certificado No: 200902779

\begin{abstract}
A Editora Conhecimento Livre, registrada no CNPJ 31.482.511/0001-10, com o prefixo ISBN 65-86-072 e DOI 10.37423, declara para os devidos fins, que o

trabalho intitulado abaixo foi submetido e publicado pela Editora como Capitulo 3 do livro "ENGENHARIA DE PRODUÇÃO: ALICERCE DA COMPETITIVIDADE".

Título:

"O USO DO RELATÓRIO A3 COMO FERRAMENTA DE IMPLEMENTAÇÃO DA FILOSOFIA LEAN NA GESTÃO DE EMPRESAS DO SETOR DE CONSTRUCÄO GIVIAC

Autor(es):

Jorge Luis Santos

DOI do livro: $10.37423 / 2020$.edcl107

DOI do Capitulo: $10.37423 / 200902779$

Da página 48 até a página 61 .
\end{abstract}

Marco Antônio Ferreira

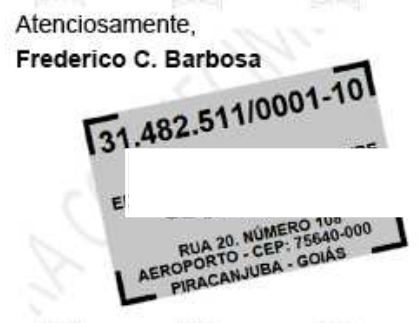

R. 20, 108 - Setor Aeroporto - Piracanjuba - GO - 75640-000

Tel. (64) 9294-7684 contato@conhecimentolivre.org | www.conhecimentolivre.org 